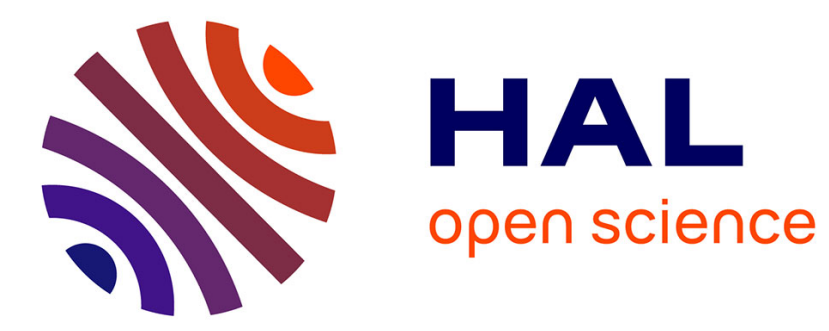

\title{
Structural changes and alkaline solubility of wood cellulose fibers after enzymatic peeling treatment
}

\author{
Nicolas Le Moigne, Kristina Jardeby, Patrick Navard
}

\section{To cite this version:}

Nicolas Le Moigne, Kristina Jardeby, Patrick Navard. Structural changes and alkaline solubility of wood cellulose fibers after enzymatic peeling treatment. Carbohydrate Polymers, 2010, 79 (2), pp.Pages 325-332. 10.1016/j.carbpol.2009.08.009 . hal-00509591

HAL Id: hal-00509591

https://hal-mines-paristech.archives-ouvertes.fr/hal-00509591

Submitted on 29 Apr 2011

HAL is a multi-disciplinary open access archive for the deposit and dissemination of scientific research documents, whether they are published or not. The documents may come from teaching and research institutions in France or abroad, or from public or private research centers.
L'archive ouverte pluridisciplinaire HAL, est destinée au dépôt et à la diffusion de documents scientifiques de niveau recherche, publiés ou non, émanant des établissements d'enseignement et de recherche français ou étrangers, des laboratoires publics ou privés. 


\section{Structural changes and alkaline solubility of wood cellulose}

\section{fibers after enzymatic peeling treatment}

N. Le Moigne, ${ }^{a}$ K. Jardeby ${ }^{b}$ and P. Navard ${ }^{a}$ *

${ }^{a}$ Mines Paris Tech, CEMEF - Centre de Mise en Forme des Matériaux, CNRS UMR 7635, BP 207, 1 rue Claude Daunesse, 06904 Sophia Antipolis Cedex, France

Member of the European Polysaccharide Network of Excellence (EPNOE), www.epnoe.eu

${ }^{\mathrm{b}}$ Borregaard Ind. Ltd., P.O. Box 162, N-1701 Sarpsborg, Norway

* Corresponding author. Tel: 33(0)493957466. Fax: 33(0)492389752.

E-Mail: patrick.navard@mines-paristech.fr

Submitted to : Carbohydrate Polymers 


\section{Abstract}

Two dissolving sulphite wood pulps were treated by an enzymatic peeling protocol and the changes in terms of structure and alkaline solubility were analyzed. The enzymatic treatment leads to a fast and large decrease of degree of polymerization and of crystallinity, showing that the enzymes do not act simply on the fiber surface. The swelling and dissolution behaviour of the treated samples showed that the enzyme mixture used has two effects at short peeling times, (i) a digestion of the primary wall which is seen by the near absence of ballooning and (ii) a destructuration action in the inside of the fiber which is seen by the large decrease of the degree of polymerization. At long peeling times, the external walls are totally digested and the fiber structure is totally destructured, as seen by the absence of birefringence. The alkaline solubility of the different treated samples was investigated in a $\mathrm{NaOH} 8 \%$ - water solution. As expected from thermodynamic considerations, there is a direct correlation between the solubility and the degree of polymerization. However, aside thermodynamics, the removal of the external walls and the macrostructural destructuration of the fibers are key factors in the improvement of the dissolution of wood cellulose fibers. At constant intrinsic viscosities of the cellulose materials, the alkaline solubility is almost two times higher when the external walls are removed. The macrostructural destructuration of fibers by enzymes allows preserving a high degree of polymerization while keeping a good alkaline solubility.

Keywords: cellulose, wood fiber, enzymatic peeling, dissolution, solubility, morphology 


\section{Introduction}

Cellulose fibers from wood and higher plants are very complex composite biomaterials. Their original structure is generally described at three length scales or levels, (i) the molecular level, (ii) the aggregated level and (iii) the macrostructural level (Klemm et al., 1998; Krässig, 1993). Except in raw wood, some plant fibers and cotton hairs, cellulose cannot be used without extraction. In case of uses other than paper and reinforcement fibers, cellulose must be dissolved since it cannot be melted. The goal that faced generations of scientists and numerous companies is to find a cheap, simple and non polluting dissolving process. The dissolution of cellulose has been investigated in many solvents over the last hundred years. However, some of the mechanisms involved in the dissolution at the different structural levels of cellulose fibers are still not well understood. In parallel, several physical and chemical methods, called "activation", have been developed to ease the dissolution of cellulose by an increase accessibility of the solvent to the whole structure, e.g. ball or vibration milling (Klemm et al., 1998; Wormald et al., 1996), steam explosion (Yamashiki et al., 1990), electron beam irradiation (Dubey et al., 2004; Fischer et al., 1985). The challenge is to be able to disrupt the aggregated and the macro structure of cellulose while preserving the original molecular solid-state. However, aside improving the dissolution, these activations are always accompanied of strong degradations of the cellulose polymer leading to low degree of polymerization (DP) values. In a recent work, it was shown that there is a restricted dissolution capacity when a cotton hair or a regenerated fiber immersed into a moderate quality solvent was put under tension and prevented to contract despite reaching sufficient swelling ratio for accessibility (Le Moigne, 2008). This result shows that it is not enough for the cellulose chains to be close to the reagent or to be not crystalline. Chains must be allowed to perform local conformational movements requiring long range chain mobility. Such a long range disruption was already suggested by Isogaï \& Attala (1998) when studying the dissolution of cellulose in aqueous $\mathrm{NaOH}$ solutions. 
The chemical environment of the cellulose chains is also playing a role in the dissolution capacity (Le Moigne, 2008). Cellulose chains of similar molecular mass can be soluble or not depending on their chemical environment and their localization in the cell wall. In a recent paper, the existence of a centripetal radial gradient in the dissolution capacity within the fiber was proposed (Le Moigne et al., 2008). The older was the deposition, the more difficult it is to dissolve it. These results were related to age-dependent structural re-organizations in the cell wall layers or more probably to the presence of non-cellulosic components around cellulose chains.

Since the presence of the primary wall is a handicap for dissolving cellulose fibers, it is important to investigate in details the influence of the removal of the external walls of wood pulp fibers on dissolution. This can be performed by an enzymatic peeling treatment. The hope is to remove only what impedes the dissolution of cellulose without degrading too much the rest of the fiber. The consequence of an enzymatic treatment on cellulose dissolution has been studied in several works. It was always suggested that the increase of solubility after an enzymatic treatment is due to a decrease of both DP and hydrogen bond density (Cao \& Huimin, 2006; Rahkamo et al., 1996). However, no data on the influence of potential macrostructural changes like removal of external layer exists. The aim of the present study is to investigate the role that macrostructural changes occurring during an enzymatic peeling treatment play on dissolution capacity of wood cellulose fibers, in addition to the classical thermodynamic parameters. 


\section{Materials and methods}

\subsection{Cellulose samples}

Three dissolving sulphite wood pulp samples were used, named VHV-S, SA (Super Aceta) and LV-U (bleached sulphite pulp) all provided by Borregaard (Norway). The main characteristics of the pulps are reported in Table 1. VHV-S and SA pulps have high intrinsic viscosity values. However, the original structure of the fiber is supposed to be more preserved in the case of the VHV-S pulp since it was digested at a higher kappa number than the SA and LV-U pulps.

\subsection{Enzymatic peeling protocol}

The enzymatic peeling protocol was adapted from the work of Sjöberg et al. (2005). This protocol was originally designed to perform a peeling of cellulosic fiber materials to determine their cross-sectional carbohydrate composition. A mixture of two commercial enzymes was used : Celluclast 1.5L (cellulase derived from Trichoderma reesei, sold by Novozymes, Denmark, used classically for the hydrolysis of lignocellulosic biomass feedstocks. This enzyme contains a broad spectrum of cellulolytic enzyme activities, like cellobiohydrolases and endo-1,4-glucanases) and Econase HC400 (highly concentrated liquid formulation of endo-1.4- beta-xylanase produced by Trichoderma reesei and standardised to minimum enzyme activity of 400,000 BXU/g as well as side activities like beta -glucanase, sold by AB enzymes, Finland).

The treatments were performed only on SA and VHV-S pulps as follows: $50 \mathrm{~g}$ of pulp (wet, never dried with a pulp consistency of approximately $16 \%$, i.e. $8 \mathrm{~g}$ of dried pulp) were mixed in $500 \mathrm{ml}$ buffer solution for a few minutes before adding the enzymes mixture. Stirring was done with a large magnet stirrer $(300 \mathrm{rpm})$ and the temperature was adjusted to $40^{\circ} \mathrm{C}$. The buffer solution was prepared as follows: $9.92 \mathrm{~g}$ NaAc was dissolved in $3.5 \mathrm{~L}$ deionised water. $\mathrm{pH}$ was adjusted to 4.0 with acetic acid. The solution was then diluted to $4 \mathrm{~L}$. The enzymes mixture was 
made of $8.35 \mathrm{ml}$ of Celluclast 1.5L and $4.20 \mathrm{ml}$ of Econase $\mathrm{HC} 400$. A fresh enzymes mixture was used for each treatment.

The peeling time was varied from 1 to $30 \mathrm{~min}$. After the peeling reaction was finished, the pulp was filtered and washed on a filter paper circle (589/1, diameter $125 \mathrm{~mm}$, ash free, black ribbon, Schleicher and Schuell MicroScience). The washing proceeded until neutral $\mathrm{pH}$ of the filtrate was measured. The samples were then dried overnight at room temperature which leads to a standard pulp consistency of $95 \%$.

\subsection{Sample weight evolution during the enzymatic peeling}

The weight of the samples was measured after the enzymatic treatment by a gravimetric method. The sample weight (\%) is defined as the amount of fibers (w/w) which remains after the enzymatic treatment, e.g. $7 \mathrm{~g}$ of pulp after treatment (pulp consistency 95\%, i.e. $6.65 \mathrm{~g}$ of dried pulp) correspond to a sample weight of $83 \%$ as regard to the initial $8 \mathrm{~g}$ of pulp introduced.

\subsection{Intrinsic viscosity and degree of polymerization}

The intrinsic viscosity was measured by Borregaard for each samples according to ISO 5351/1B (determination of the intrinsic viscosity of pulp in a dilute cupri-ethylenediamine (CuEn) solution). The intrinsic viscosity is almost linearly correlated with DP and can be calculated by the following formula (Immergut et al., 1953): $\mathrm{DP}^{0.905}=0.805[\eta](\mathrm{ml} / \mathrm{g})$.

\subsection{Crystallinity and carbohydrate composition}

The crystallinity was measured by FTIR-ATR. This indirect method was previously calibrated by wide angle X-ray scattering and by ${ }^{13} \mathrm{C}-\mathrm{NMR}$ (Baldinger et al., 2000) such as the evaluation and correlation of IR-spectra from various samples fully characterized by wide angle X-ray scattering and ${ }^{13} \mathrm{C}-\mathrm{NMR}$ provide algorithms to determine the degree of crystallinity from the 
FTIR-ATR measurements. It was indeed demonstrated earlier by Nelson \& O'Connor (1964) that a linear correlation can be found between the IR ratio determined by FTIR and the crystallinity determined by X-ray diffraction. A recent study performed within the European Polysaccharide Network of Excellence (EPNOE) confirmed the fact that the FTIR-ATR method gives reliable results when compared to other methods (EPNOE, unpublished results).

The carbohydrate composition was determined by HPLC (anion chromatography with pulsed amperometric detection) after a two step sulfuric acid hydrolysis. Both crystallinity and carbohydrate composition analyses were performed by Lenzing AG (Austria).

\subsection{Swelling and dissolution observations}

The swelling and dissolution observations were performed in two solvent systems:

- $\quad N$-methylmorpholine- $N$-oxide (NMMO) with $22 \%$ of distilled water (w/w), i.e. NMMO$22 \%$ water : $0.78 \mathrm{~g}$ of NMMO with $0.22 \mathrm{~g}$ of distilled water for a $1 \mathrm{~g}$ mixture. The NMMO was provided by Sigma-Aldrich.

- $8 \%$ of $\mathrm{NaOH}$ in distilled water (w/w), i.e. $\mathrm{NaOH} 8 \%$ - water : $0.08 \mathrm{~g}$ of $\mathrm{NaOH}$ and $0.92 \mathrm{~g}$ of distilled water for a $1 \mathrm{~g}$ mixture. The $\mathrm{NaOH}$ was provided by Sigma-Aldrich.

Samples were observed between two glass plates by optical microscopy in transmission mode with a Metallux 3 (Leitz) equipped with a high resolution 3-CCD camera JVC KY-F75U and a Linkam TMS 91 stage settled at $90^{\circ} \mathrm{C}$ for the NMMO solvent system and at $-6^{\circ} \mathrm{C}$ for the $\mathrm{NaOH}$ solvent system. The solvent contained in a pipette was injected by capillarity between the two glass plates. No agitation was applied to the system. 


\subsection{Pulp solution preparation}

The non-treated and treated pulps were dissolved in a $\mathrm{NaOH} 8 \%$ - water solution to test their alkaline solubility. The solutions were prepared as follows: $132 \mathrm{~g}$ of $\mathrm{NaOH} 12 \%$ - water were stored at $-6^{\circ} \mathrm{C} .2 \mathrm{~g}$ of pulp were added to $66 \mathrm{~g}$ of distilled water and stored 1 hour at $4^{\circ} \mathrm{C}$. Then, $\mathrm{NaOH} 12 \%$ - water and cellulose - water solutions were mixed together during two hours at $-6^{\circ} \mathrm{C}$ and $1000 \mathrm{rpm}$ giving $200 \mathrm{~g}$ of a solution of $1 \%$ cellulose in $\mathrm{NaOH} 8 \%$ - water. These $1 \%$ cellulose solutions were directly centrifuged to isolate the insoluble fraction.

\subsection{Isolation by centrifugation of the insoluble fractions}

Centrifugation was performed on a Hettich Universal 320RHK centrifuge equipped with a $1620 \mathrm{~A}$ rotor and a Julabo cryostat. The centrifuge was settled at $9000 \mathrm{rpm}(9050 \mathrm{~g})$ and the centrifugation time was 5 minutes. The centrifuge tubes were refrigerated at $4^{\circ} \mathrm{c}$ before starting the centrifugation protocol. Despite the cryostat of the centrifuge was set at $0^{\circ} \mathrm{C}$, the temperature inside the rotating sample increased up to 8 to $12^{\circ} \mathrm{C}$ due to centrifugation heating and heat loss between the cryostat and the centrifuge. The low concentration of cellulose (1\%), the low temperature (below $12^{\circ} \mathrm{C}$ ) and the short centrifugation time $(5 \mathrm{~min})$ prevent gelation of the samples during centrifugation (Roy et al., 2003). At the end of the centrifugation protocol, we obtained a centrifugation pellet (the insoluble fraction) and a supernatant being the soluble fraction which is transparent when observed by optical microscopy.

\subsection{Optical microscopy observations of the insoluble fractions}

After centrifugation, insoluble fractions were observed between two glass plates by optical microscopy in transmission mode with and without cross-polarizer with a Metallux 3 (Leitz) equipped with a high resolution 3-CCD camera JVC KY-F75U. 


\subsection{Amount of insoluble material and alkaline solubility}

The insoluble fractions were washed in distilled water in several baths until the $\mathrm{pH}$ was neutral. The resulting products were dried overnight in an air oven at $50^{\circ} \mathrm{C}$. Each fraction was weighed after total drying. The amount of insoluble fraction was defined as the following weight ratio: [Dried insoluble fraction] / [Initial dried sample introduced]. The alkaline solubility is thus defined as the inverse of the fraction of insoluble material amount. The measured pulp consistency of the samples introduced in the $\mathrm{NaOH} 8 \%$ - water solution was $96 \%$ (after drying in the same conditions as the insoluble fractions, i.e. $50^{\circ} \mathrm{C}$ in an air oven, overnight), i.e. the initial 2 $\mathrm{g}$ of sample introduced corresponds to $1.92 \mathrm{~g}$ of dried sample.

\section{Results and discussion}

\subsection{Molecular structural changes after the enzymatic peeling treatment}

The enzymes mixture is made of Celluclast 1.5L and Econase HC 400. As described by Ramos et al. (1999) and Rosgaard et al. (2007), the Celluclast 1.5L contains a large spectrum of enzymes, most notably cellobiohydrolases (CBHs) and endo-1,4- $\beta$-glucanase (EGs). These two enzymes are able to digest cellulose to give cellobiose and glucose. It is known that the EGs digest preferentially the easily available amorphous chains and that the $\mathrm{CBHs}$ are able to degrade crystalline zones (Henrissat et al., 1985). In addition, CBHs are able to hydrolyze cellulose at both reducing and non-reducing ends by its two activities, CBHI and CBHII respectively (Divne et al., 1994; Rosgaard et al., 2007; Rouvinen et al., 1990). The degradation of the amorphous zones by the EGs makes entrance points for the CBHs to attack crystalline cellulose (Lynd et al., 2002). This concerted action of CBHs with EGs is a synergism phenomenon that has been reported on both lignocellulosic substrates and pure cellulose substrates for varying cellulase systems (Gusakov et al., 2007; Mansfield \& Meder, 2003; Rosgaard et al., 2007; Zhang \& Lynd, 2004). Amorphous zones were also shown to degrade much faster than crystalline zones (Lynd et al., 
2002; Zhang \& Lynd, 2004). Crystallinity should thus increase during cellulose hydrolysis. However, due to varying and conflicting results on the change of crystallinity during hydrolysis, crystallinity is not considered as a determinant parameter to analyze the rate of enzymatic hydrolysis (Zhang \& Lynd, 2004). The Econase HC 400 is an endo-1,4- $\beta$-xylanase which attacks specifically xylan, i.e. arabinoglucoronoxylan, on interior portions of the chains to give xylose, arabinose and 4-O-MeGlcA.

Figure 1 shows that the sample weight decreases continuously for both VHV-S and SA pulps but much faster during the first five minutes. It means that the activity of the enzymes is effective but not constant all along the 30 minutes of peeling. The evolution of the intrinsic viscosity (Figure 2), which can be almost linearly correlated with the DP (see Materials and methods), shows a large and fast decrease during the first five minutes before reaching a plateau at nearly $600 \mathrm{ml} / \mathrm{g}$ and $400 \mathrm{ml} / \mathrm{g}$ for VHV-S and SA respectively. The corresponding DP values are 925 and 590 for VHV-S and SA respectively (2375 and 1410 initially) meaning that cellulose chains were cut 2.4 to 2.6 times on average. The first fast decrease phase can be attributed to the action of the EGs which act on less ordered regions and interior portions of the chain and thus rapidly decrease the DP. Then, the CBHs, which act only on chain ends, decrease the DP only incrementally. These results are indeed supported by several studies which are listed in the well documented review of Zhang \& Lynd (2004). This large decrease of DP should not be observed if the enzyme activity was localized only at the surface of the fibers. For a sample weight of $70 \%$, i.e. $30 \%$ of the initial weight removed, the intrinsic viscosity is decreased by more than two times for both SA and VHV-S pulp (Figure 3). The concept of peeling as enzymes digest the fibers layer by layer is thus not realistic. There is either a gradient of digestion inside the fiber or digestions deep inside the fiber. A gradient of digestion is understandable if the strong degradation of one layer is allowing enzymes to move more deeply inside cell walls, creating a degradation gradient. Another option is that enzymes are moving inside the wall through available pores and voids. In any case, 
the enzymes are active both in the outside and the inside of the fibers. Crystallinity follows the same evolution as the sample weight and the intrinsic viscosity. A large decrease of almost 50\% of the initial crystallinity ratio is observed during the first five minutes, followed by a slow decrease (Figure 4).

The activity of the enzymes mixture is thus very high during the first five minutes of treatment with nearly 2.5 cuts of the cellulose chains on average. The digestion occurs by two simultaneous mechanisms: (i) the digestion of the amorphous phase by the EGs which decrease the intrinsic viscosity but does not decrease the crystallinity, (ii) the digestion of the crystalline phase by the CBHs which decrease both the intrinsic viscosity and the crystallinity. It has to be noticed that some side effect of the enzymatic hydrolysis could also have an influence on the lower activity of the enzymes after the first five minutes of treatment. In fact, an inhibition of the enzymes by the hydrolysis products, i.e. cellobiose and glucose, can happen depending of the accumulation of this product in the microenvironments in which hydrolysis occurs (Lynd et al., 2002).

\subsection{Influence of the enzymatic peeling treatment on the macrostructural morphology}

The trends of the intrinsic viscosity and the crystallinity have shown that the enzymes attack both the outside and the inside of the fibers. However, these results give no information about the effect of the enzymes on the fiber macrostructure. To evaluate in what extent the macrostructural morphology have been affected by the treatment, we have analyzed and compared the swelling and dissolution mechanisms of non-treated and treated samples between two glass plates without agitation. The solvent quality was adjusted to be in the zone where the ballooning phenomenon occurs, i.e. NMMO with 19 to $24 \%$ of water and $\mathrm{NaOH} 8 \%$ - water (Cuissinat \& Navard, 2006a, b). In the ballooning zone, it is possible to clearly identify the behaviour of the three main walls of wood and cotton fibers (Le Moigne et al., 2008), i.e the primary wall, the S1 wall and the S2 wall. In NMMO - 19 to $24 \%$ water, the inside of the fiber (S2 wall) fragments and dissolves, while the 
S1 wall swells significantly to form the membrane of the balloons and the primary wall breaks and rolls up to form unswollen sections and helices. Then, the S1 wall and the primary wall dissolve slowly. In $\mathrm{NaOH}$-water system, no fragmentation of the $\mathrm{S} 2$ wall is observed but the large swelling of the secondary wall leads to the breaking of the primary wall which also forms helices and unswollen sections. Then, the fibers stay in this ballooned state if no agitation or shearing is applied to the system (Le Moigne, 2008).

If the external walls - the primary wall and the S1 wall - are removed, unswollen sections, helices and membranes should not be observed. Only fragmentation should be observed in the case of NMMO-water system and only a large homogeneous swelling should be observed in the case of $\mathrm{NaOH}$-water system. This hypothesis was checked. First non-treated fibers were observed. As can be seen on Figures 5a, 5d, 6a and 6d, non-treated pulps show a ballooning phenomenon meaning that the external walls were present, despite of the difference of sulphite treatment severity and intrinsic viscosity values (1430 ml/g for VHV-S and $880 \mathrm{ml} / \mathrm{g}$ for SA). The original wall structure is thus initially present in both VHV-S and SA samples.

After a short peeling time of around $3 \mathrm{~min}$, the ballooning phenomenon almost completely disappears. Only fragmentation and some membrane parts are observable in NMMO- 22\% water, as seen in Figures 5b and 5e. In $\mathrm{NaOH} 8 \%$ - water, fibers are largely swollen (100 $\mu \mathrm{m}$ in diameter) without helices and unswollen sections, as seen in Figure 6b. A few ballooned fibers are still observable (Figures 5b, 5e, 6b) showing that the enzymatic treatment was not fully homogeneous. The external walls, especially the primary wall, have thus been removed after a short peeling time which is in accordance with the results of Sjöberg (2002) obtained with the same enzymes mixture.

At longer peeling time, fibers dissolve by fragmentation in NMMO - $22 \%$ water (Figures $5 \mathrm{c}$ and 5f) and swell dramatically (almost $200 \mu \mathrm{m}$ ) in $\mathrm{NaOH} 8 \%$ - water (Figures 6c and 6e) meaning that the external walls have been totally removed and that the structure of the fiber has been very 
damaged by the enzymatic hydrolysis. Moreover, there is no visible birefringence of the highly swollen fibers and sections observed in $\mathrm{NaOH} 8 \%$ - water (Figure 6f) which indicates that the crystalline zones have entirely disappeared (or at least are of such small size that they do not generate a visible birefringence) and that all orientational order has disappeared.

The enzyme mixture used has thus two effects at short peeling times, (i) a digestion of the primary wall which is seen by the near absence of ballooning and (ii) a destructuration action in the inside of the fiber which is seen by the large decrease of DP. At long peeling time, the external walls are totally digested and the fiber structure is totally destructured, as seen by the absence of birefringence.

\subsection{Observations and quantification of insoluble material in $\mathrm{NaOH}$ - water system}

The different samples were dissolved in $\mathrm{NaOH} 8 \%$ - water as described in the Materials and methods. The resulting solutions were centrifuged to isolate the insoluble material fractions. We showed in a recent work that the dissolution of wood fibers in $\mathrm{NaOH} 8 \%$ - water solutions occurs by successive dismantlement and fragmentation mechanisms mainly governed by the high swelling and the shearing involved during the solution preparation (Le Moigne, 2008). The different steps of dissolution finally lead to a soluble fraction which is transparent when observed by optical microscopy.

The observation by optical microscopy of the insoluble material clearly shows the two effects of the enzymatic peeling treatment, i.e. the removal of the external walls and the destructuration of the fiber. Non-treated samples mostly stay in a low swollen or ballooned state even after two hours of mixing (Figures 7a and 7d). The SA sample contains fibers with high swelling ratio which can be related to the more severe digestion of the fibers during the original sulphite pretreatment. Several fibers are cut in sections due to the shearing involved by the mixing and few fragments can also be observed. After 2 min of peeling, the shape of the insoluble parts is 
changing drastically. Only highly swollen sections and flat rings are observed (Figures 7b and 7e). The removal of the primary wall by the enzymatic peeling gives a large swelling of the fibers and a subsequent cutting in flat rings. This cutting in flat rings was observed when putting and submitting fibers to shearing in $\mathrm{NaOH} 8 \%$ - water, independently of the origin and the preparation mode of the wood pulp. With fibers without primary wall, the large swelling was shown to be sufficient to dismantle the fibers in highly swollen sections and flat rings (Le Moigne, 2008). At longer peeling times, the insoluble material is made of small fragments implying that the fibers were totally destructured by the enzyme treatment (Figures $7 \mathrm{c}$ and $7 \mathrm{f}$ ).

The evolution of the amount of insoluble material as a function of peeling time is divided in two main phases (Figure 8). During the first minutes of treatment, the amount of insoluble material decreases quickly. Then it decreases very slowly and the alkaline solubility reaches a plateau. The evolution of the alkaline solubility is related with all the structural changes that occurred upon the enzymatic peeling treatment.

\subsection{Relationship between the structural changes and the alkaline solubility}

As can be seen on Figure 9, the amount of insoluble material follows a linear relationship as a function of intrinsic viscosity and crystallinity. A simple way to explain the evolution of the alkaline solubility after the enzymatic treatment is thus to associate the increase of solubility to the decrease of the DP and the crystallinity. This interpretation is supported by several studies (Cao \& Huimin, 2006; Rahkamo et al., 1996).

However, the morphological study of the insoluble parts gives a more comprehensive interpretation on the influence of the structural changes due to the enzymatic treatment on the alkaline solubility. Figure 10 gives the amount of insoluble material of the non-treated (A, B) and 2 min enzymatically treated (C, D) SA and VHV-S pulps and of the LV-U pulp (E). By following the dotted line number 1 of Figure 10, an interesting result is found. Despite the large difference 
of intrinsic viscosity of the two original SA and VHV-S pulps $(880 \mathrm{ml} / \mathrm{g}$ and $1430 \mathrm{ml} / \mathrm{g}$ respectively), the amount of insoluble material is nearly the same. The DP is thus not the only parameter that governs the alkaline solubility after enzymatic treatment. We showed recently that even if the soluble fraction has a lower mean molar mass than the insoluble one, there is a significant fraction of cellulose chains that can be soluble or not while having the same molar mass (Le Moigne, 2008). The dotted line number 2 of Figure 10 shows that the amount of insoluble material of the treated VHV-S sample (D, black diamond) is nearly two times lower than the original SA sample (A, grey square) while keeping the same intrinsic viscosity. This observation has to be related with the removal of the external walls that occur during the first minutes of treatment. In fact, the external walls are still present in the original SA sample (Figure 7d) while they have been removed in the 2 min treated VHV-S sample (Figure 7b). The removal of the external walls allows a higher swelling and thus eases the dismantlement and the fragmentation of the fibers during the solution preparation. The dotted line 3 shows that the LV-U sample (E) which was not enzymatically treated present similar amount of insoluble material as compared to the treated SA and VHV-S samples (C, D) while its intrinsic viscosity is more than two times lower. The enzyme destructuration allows to reach the same dissolution capacity, i.e. the same insoluble material amount, at higher molecular weights by favoring the fragmentation and the dissolution of the fibers.

The removal of the external walls and the macrostructural destructuration of the fibers must thus be considered as key factors in the dissolution capacity of wood fibers in $\mathrm{NaOH} 8 \%$ - water.

\section{Conclusions}

The enzymatic peeling treatment used in this study is leading to important structural changes on the two treated wood pulps. Aside decreasing DP and crystallinity, the enzymes mixture is able to remove the external walls and to destructure the fibers at the macrostructural level. The action 
of the enzymes and the associated products after the dissolution of the treated samples in $\mathrm{NaOH}$ $8 \%$ - water are schematically represented in Figure 11.

Beyond the classical thermodynamic considerations evocated for the increase of solubility after enzymatic treatment, we have shown that the removal of the external walls and the macrostructural destructuration of the fiber are key factors in the dissolution capacity of wood cellulose fibers. In fact, these morphological changes ease the swelling and the breaking of the fibers into fragments in $\mathrm{NaOH} 8 \%$ - water solutions and thus favor dissolution. These results are supported by a recent study showing that the macrostructural destructuration involved by steam explosion or acidic hydrolysis also leads to the same dissolution mechanisms (Le Moigne, 2008). The microenvironment around the cellulose chains, e.g. the hemicelluloses complex, is also an important parameter to consider for the dissolution of cellulose fiber since the insoluble materials contain more hemicelluloses than the soluble fraction (Le Moigne, 2008).

\section{Acknowledgements}

The authors thank Borregaard, Dow Wolff Cellulosics GmbH, Lenzing AG and Spontex for their financial and technical support and their staff for scientific discussions.

\section{References}

Baldinger, T., Moosbauer, J. \& Sixta, H. (2000). Supermolecular structure of cellulosic materials by Fourier transform infrared spectroscopy (FT-IR) calibrated by WAXS and ${ }^{13} \mathrm{C}$ NMR. Lenzinger Berichte, 79, 15-17

Cao, Y. \& Huimin, T. (2006). Improvement of alkali solubility of cellulose with enzymatic treatment. Applied Microbiology and Biotechnology, 70, 176-182. 
Cuissinat, C. \& Navard, P. (2006a). Swelling and Dissolution of Cellulose Part 1: Free Floating Cotton and Wood Fibres in $N$-Methylmorpholine- $N$-oxide-Water Mixtures. Macromolecular Symposia, 244, 1-18.

Cuissinat, C. \& Navard, P. (2006b). Swelling and Dissolution of Cellulose Part II: Free Floating Cotton and Wood Fibres in NaOH-Water-Additives Systems. Macromolecular Symposia, 244, 19-30.

Divne, C., Ståhlberg, J., Reinikainen, T., Ruohonen, L., Pettersson, G., Knowles, J. K., Teeri, T. T. \& Jones, T. A. (1994). The three-dimensional crystal structure of the catalytic core of cellobiohydrolase I from Trichoderma reesei. Science, 265, 524-528.

Dubey, K. A.,. Pujari, P. K., Ramnani, S. P., Kadam, R. M. \& Sabharwal, S. (2004). Microstructural studies of electron beam-irradiated cellulose pulp. Radiation Physics and Chemistry, 69, 395-400.

Fischer, K., Goldberg, W. \& Wilke, M. (1985). Strahlvorbehandlung von zellstoff für die regeneratfaserherstellung. Lenzinger Berichte, 59, 32-39.

Gusakov, A. V., Salanovich, T. N., Antonov, A. I., Ustinov, B. B., Okunev, O. N., Burlingame, R., Emalfarb, M., Baez, M. \& Sinitsyn, A. P. (2007). Design of highly efficient cellulase mixtures for enzymatic hydrolysis of cellulose. Biotechnology and Bioengineering, 97, 1028-1038.

Henrissat, B., Driguez, H., Viet, C. \& Schülein, M. (1985). Synergism of cellulases from Trichoderma reesei in the degradation of cellulose. Nature Biotechnology, 3, 722-726.

Immergut, E. H., Schurz, J., \& Mark, H. (1953). Viskositätzahl-molekulargewichts-beziehung für cellulose und untersuchungen von nitrocellulose in verschiedenen lösungmitteln. Monatshefte für Chemie, 84, 219-249.

Isogai, A. \& Atalla, R. H. (1998). Dissolution of cellulose in aqueous NaOH solutions. Cellulose, 5, 309-319. 
Klemm, D., Philipp, B., Heinze, T., Heinze, U. \& Wagenknecht, W. (1998). Comprehensive Cellulose Chemistry (Vol 1). Weinheim: Wiley-VCH.

Krässig, H. A. (1993). M.B. Huglin (Eds.). Cellulose - Structure, Accessibility and Reactivity. Polymer Monographs (Vol. 11). Amsterdam: Gordon and Breach Science Publishers.

Le Moigne, N. (2008). Swelling and dissolution mechanisms of cellulose fibres. Ph.D. dissertation, Ecole National Supérieure des Mines de Paris, Sophia Antipolis, France. http://pastel.paristech.org/4570/

Le Moigne, N., Montes, E., Pannetier, C., Höfte, H. \& Navard, P. (2008). Gradient in dissolution capacity of successively deposited cell wall layers in cotton fibres. Macromolecular Symposia, 262, 1, 65-71.

Lynd, L. R., Weimer, P. J., Van Zyl, W. H. \& Pretorius, I. S. (2002). Microbial cellulose utilization: fundamentals and biotechnology. Microbiology and Molecular Biology Reviews, $66,506-577$.

Mansfield, S. D. \& Meder, R. (2003). Cellulose hydrolysis - the role of monocomponent cellulases in crystalline cellulose degradation. Cellulose, 10, 159-169.

Nelson, M. L. \& O'Connor, R. T. (1964). Relation of certain infrared bands to cellulose crystallinity and crystal lattice type. Part II. A new infrared ratio for estimation of crystallinity in celluloses I and II. Journal of Applied Polymer Science, 8, 1325-1341.

Rahkamo, L., Siika-Aho, M., Vehviläinen, M., Dolk, M., Viikari, L., Pertti, N. \& Buchert, J. (1996). Modification of hardwood dissolving pulp with purified Trichoderma reesei cellulases. Cellulose, 3, 153-163.

Ramos, L. P., Zandoná Filho, A., Deschamps, F. C. \& Saddler, J. N. (1999). The effect of Trichoderma cellulases on the fine structure of a bleached softwood kraft pulp. Enzyme and Microbial Technology, 24, 371-380. 
Rosgaard, L., Pedersen, S., Langston, J., Akerhielm, D., Cherry, J. R. \& Meyer, A. S. (2007). Evaluation of minimal Trichoderma reesei cellulase mixtures on differently pretreated Barley straw substrates. Biotechnology Progress, 23, 1270-1276.

Rouvinen, J., Bergfors, T., Teeri, T., Knowles, J. K. \& Jones, T. A. (1990). Three-dimensional structure of cellobiohydrolase II from Trichoderma reesei. Science, 249, 380-386.

Roy, C., Budtova, T. \& Navard, P. (2003). Rheological properties and gelation of aqueous cellulose-NaOH solutions. Biomacromolecules, 4, 259-264.

Sjöberg, J. (2002). Characterization of chemical pulp fiber surfaces with an emphasis on the hemicelluloses. Ph.D. dissertation, KTH Royal Institute of Technology, Stockholm (Sueden)

Sjöberg, J., Potthast, A., Rosenau, T., Kosma, P. \& Sixta, H. (2005). Cross-sectional analysis of the polysaccharide composition in cellulosic fiber materials by enzymatic peeling and highperformance capillary zone electrophoresis (HPCE). Biomacromolecules, 6, 3146-3151.

Wormald, P., Wickholm, K., Larsson, P. T. \& Iversen, T. (1996). Conversions between ordered and disordered cellulose. Effects of mechanical treatment followed by cyclic wetting and drying. Cellulose, 3, 141-152.

Yamashiki, T., Matsui, T., Saitoh, M., Okajima, K., Kamide, K. \& Sawada, T. (1990). Characterisation of cellulose treated by the steam explosion method. Part 1: Influence of cellulose resources on changes in morphology, degree of polymerisation, solubility and solid structure. British Polymer Journal, 22, 73-83.

Zhang, Y-H. P. \& Lynd, L. R. (2004). Toward an aggregated understanding of enzymatic hydrolysis of cellulose: non-complexed cellulase systems. Biotechnology and Bioengineering, 88, 797-824. 


\section{Figure and table Captions}

Table 1 Main characteristics of the wood pulp samples used.

Fig. 1. Evolution of the sample weight versus the peeling time for the VHV-S and SA pulps.

Fig. 2. Evolution of the intrinsic viscosity versus the peeling time for the VHV-S and SA pulps.

Fig. 3. Intrinsic viscosity / Initial intrinsic viscosity ratio versus the sample weight for the VHV-S and SA pulps.

Fig. 4. Evolution of the crystallinity versus the peeling time for the VHV-S and SA pulps.

Fig. 5. Swelling and dissolution behavior of VHV-S and SA pulps in NMMO- 22\% water. (a) Non-treated VHV-S, (b) VHV-S after 3 min of enzymatic peeling and (c) VHV-S after 10 min of enzymatic peeling; (d) Non-treated SA, (e) SA after 3 min of enzymatic peeling and (f) SA after 10 min of enzymatic peeling.

Fig. 6. Swelling and dissolution behavior of VHV-S and SA pulps in $\mathrm{NaOH} 8 \%$ - water. (a) Non-treated VHV-S, (b) VHV-S after 3 min of enzymatic peeling and (c) VHV-S after 10 min of enzymatic peeling; (d) Non-treated SA, (e) SA after 10 min of enzymatic peeling and (f) SA after 10 min of enzymatic peeling observed between cross-polarizer.

Fig. 7. Observations by optical microscopy of the insoluble material in a $\mathrm{NaOH} 8 \%$-water solution. (a) Non-treated VHV-S, (b) VHV-S after 2 min of enzymatic peeling and (c) VHV-S after 30 min of enzymatic peeling; (d) Non-treated SA, (e) SA after 2 min of enzymatic peeling and (f) SA after 30 min of enzymatic peeling.

Fig. 8. Evolution of the amount of insoluble material in $\mathrm{NaOH} 8 \%$ - water versus the peeling time for the VHV-S and SA pulps. 
Fig. 9. Relationship between the amount of insoluble material and the intrinsic viscosity (up), and the crystallinity (down) for the non-treated and treated VHV-S and SA pulps.

Fig. 10. Final amount of insoluble material versus the intrinsic viscosity for the non-treated SA and VHV-S pulps (A, B), the 2 min treated SA and VHV-S pulps (C, D) and the nontreated LV-U pulp (E).

Fig. 11. Schematic representation of the action of the enzymes on the macrostructural morphology of wood cellulose fibers and the associated dissolution products in $\mathrm{NaOH}$ $8 \%$ water. 


\section{Table 1}

\begin{tabular}{|l|l|l|c|c|c|c|c|}
\hline Pulp samples & Wood origin & Supplier & Intrinsic viscosity * & Glucan ${ }^{\circ}$ & Xylan ${ }^{\circ}$ & Mannan ${ }^{\circ}$ & \$rystallinity ${ }^{\circ}$ \\
\hline VHV-S & Norway spruce & Borregaard & $1430 \mathrm{ml} / \mathrm{g}$ & $90 \%$ & $3,5 \%$ & $1,8 \%$ & $46 \%$ \\
\hline SA & Norway spruce & Borregaard & $880 \mathrm{ml} / \mathrm{g}$ & $94,7 \%$ & $1,9 \%$ & $1,1 \%$ & $49 \%$ \\
\hline LV-U & Norway spruce & Borregaard & $350 \mathrm{ml} / \mathrm{g}$ & - & - & - & - \\
\hline
\end{tabular}

* Measured by Borregaard according to ISO 5351/1B

- Measured by Lenzing AG as described in the materials and methods 


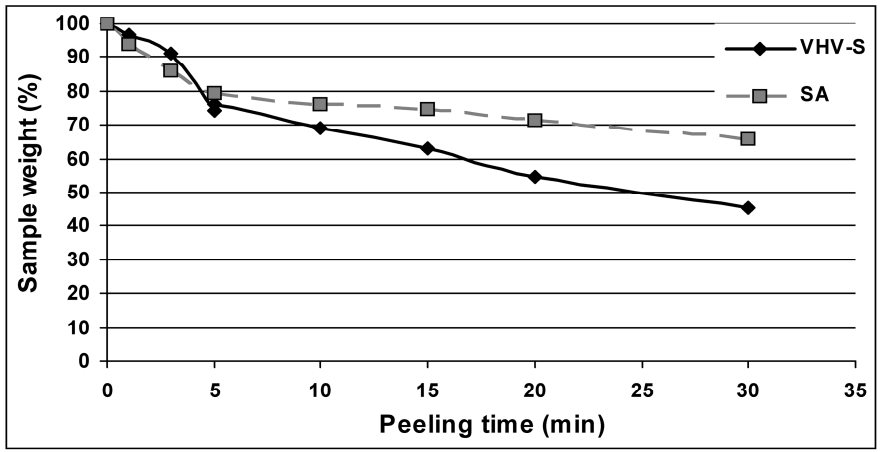

\section{Fig 1}




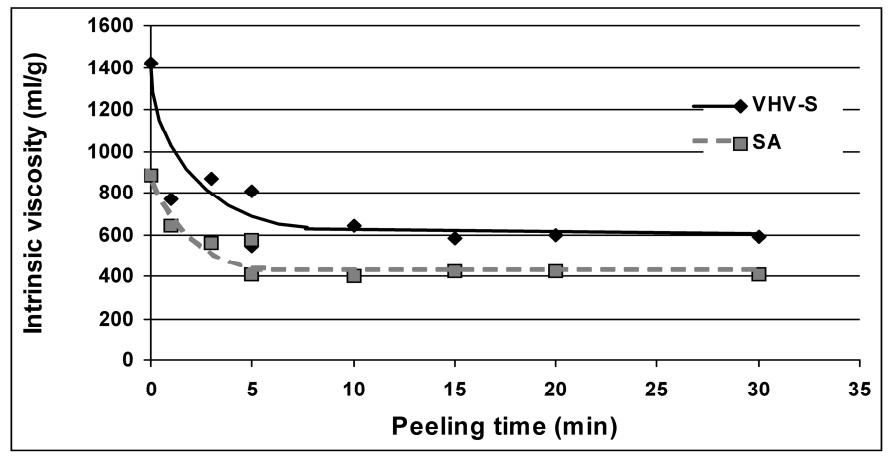

Fig 2 


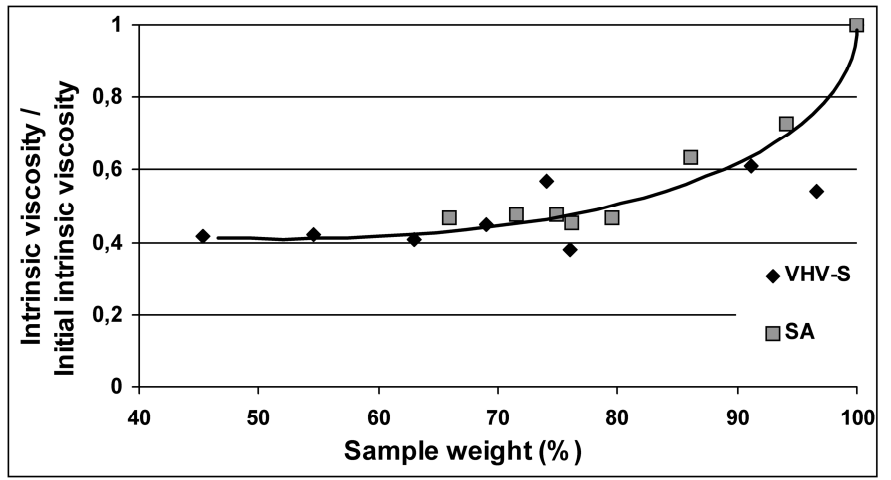

Fig 3 


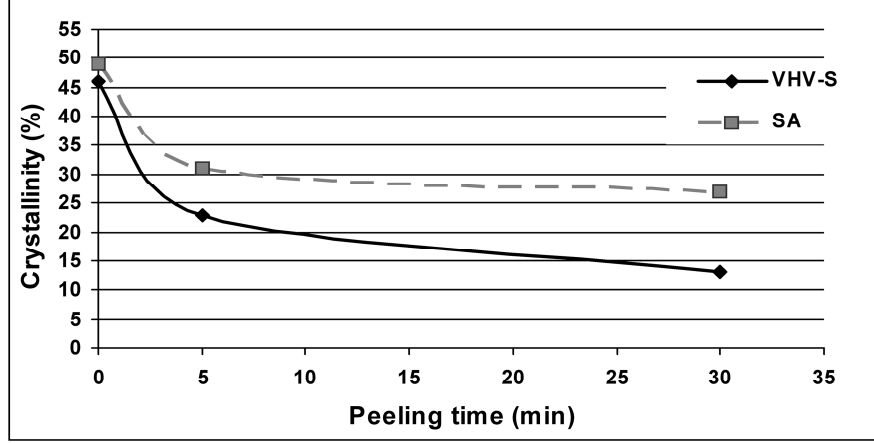

Fig 4 


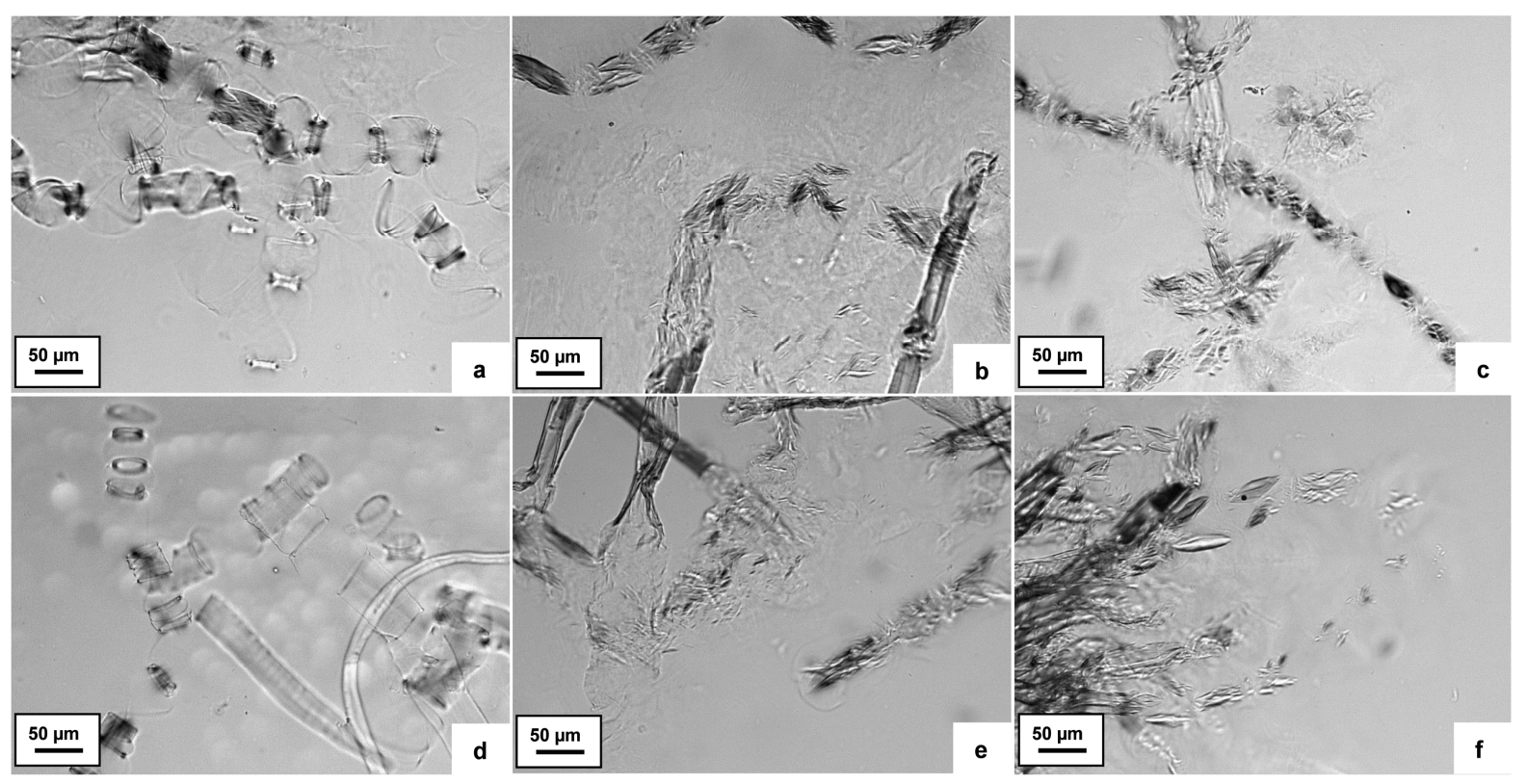

Fig 5 


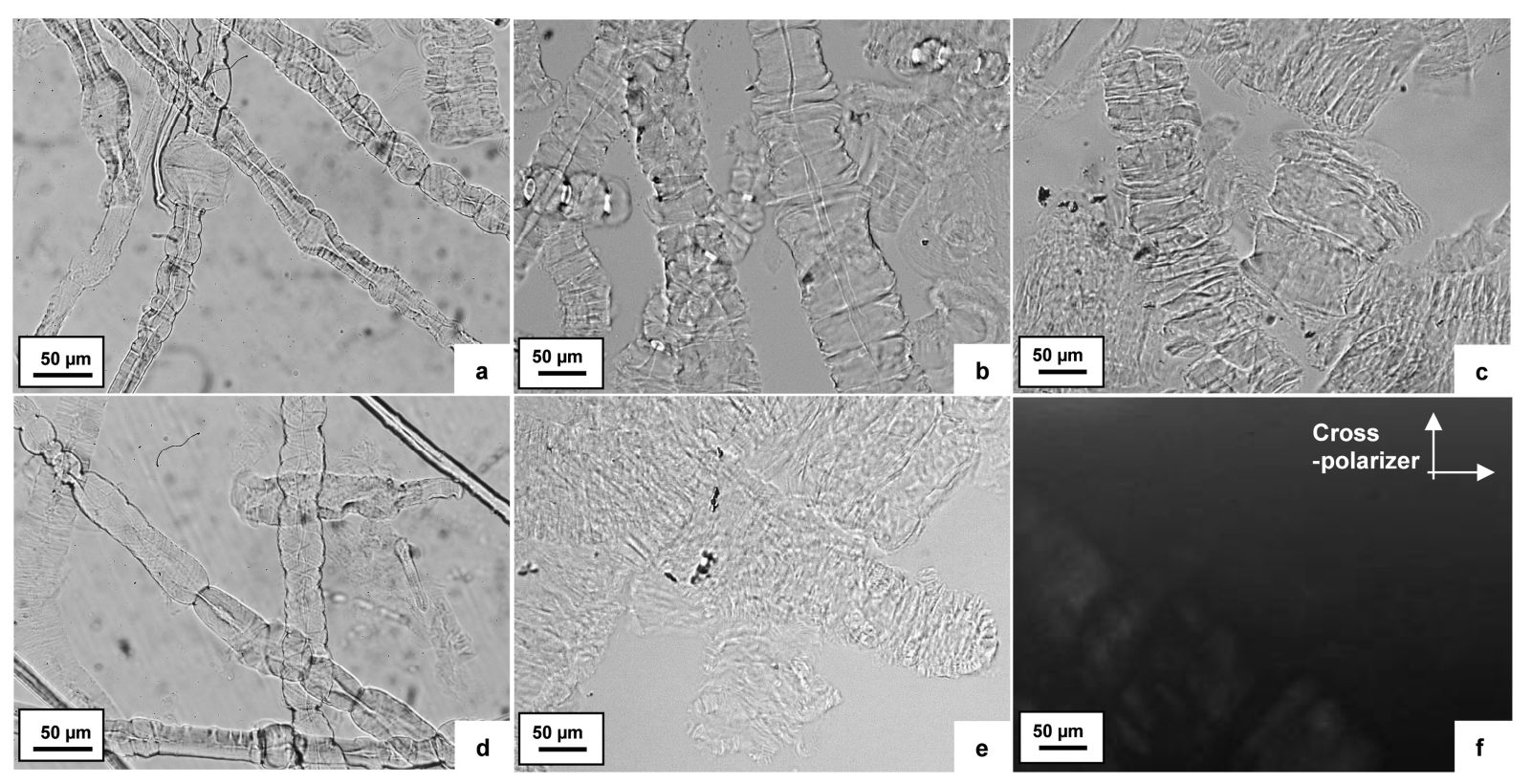

Fig 6 


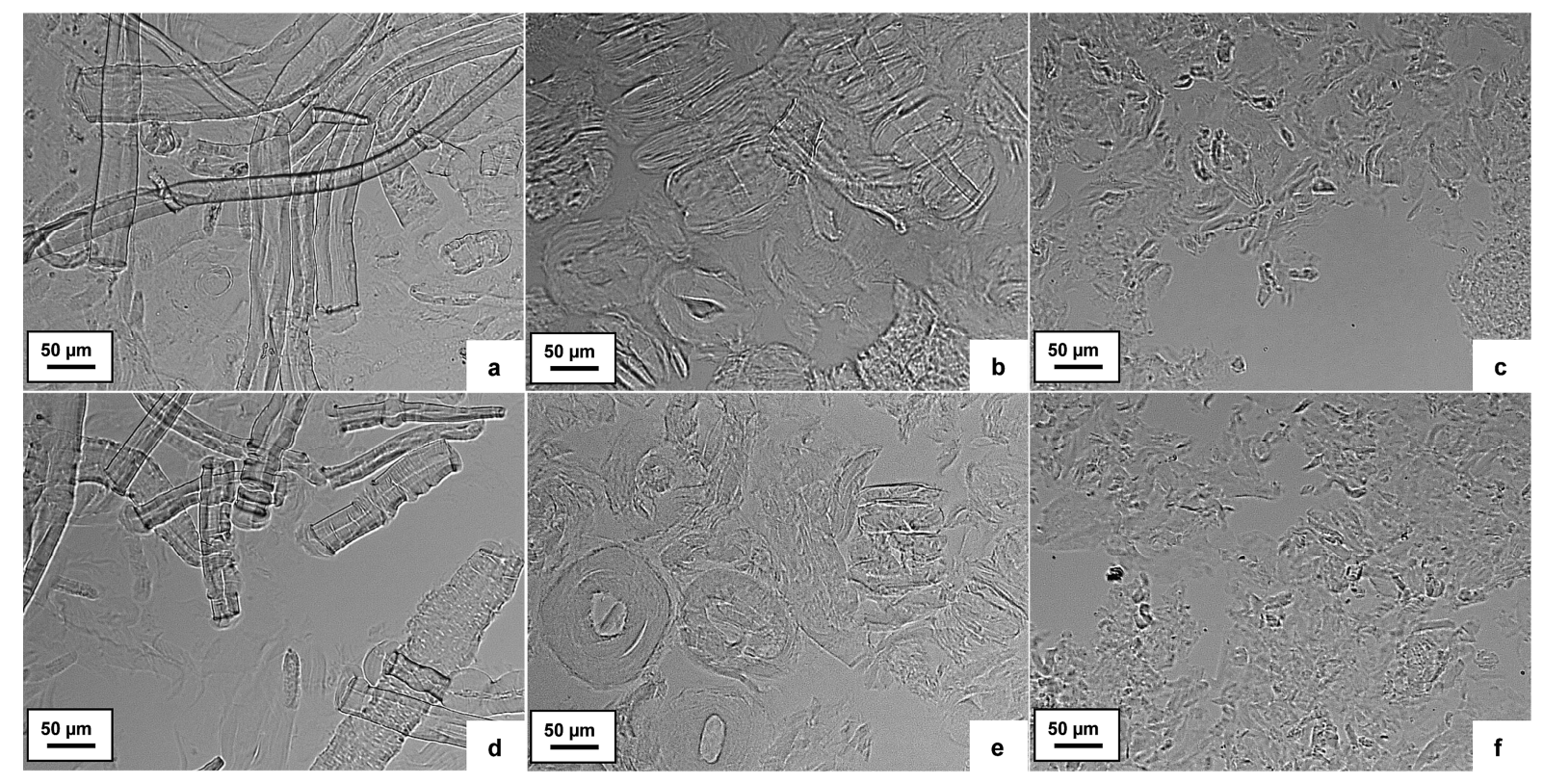

Fig 7 


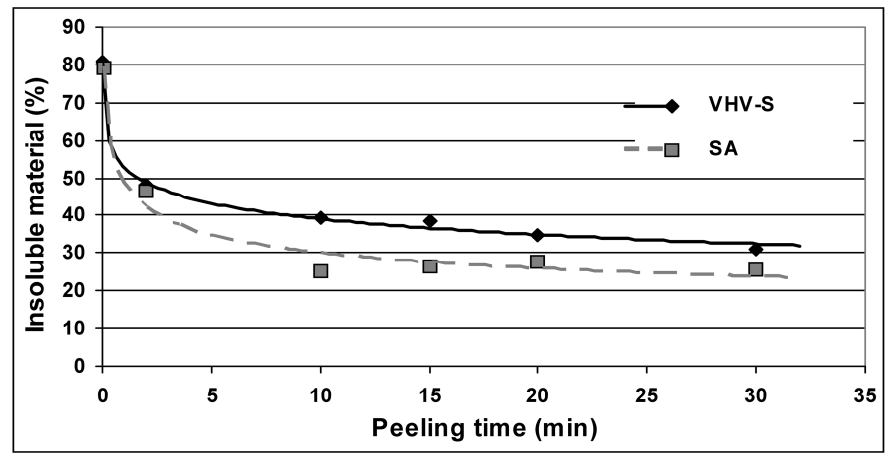

Fig 8 

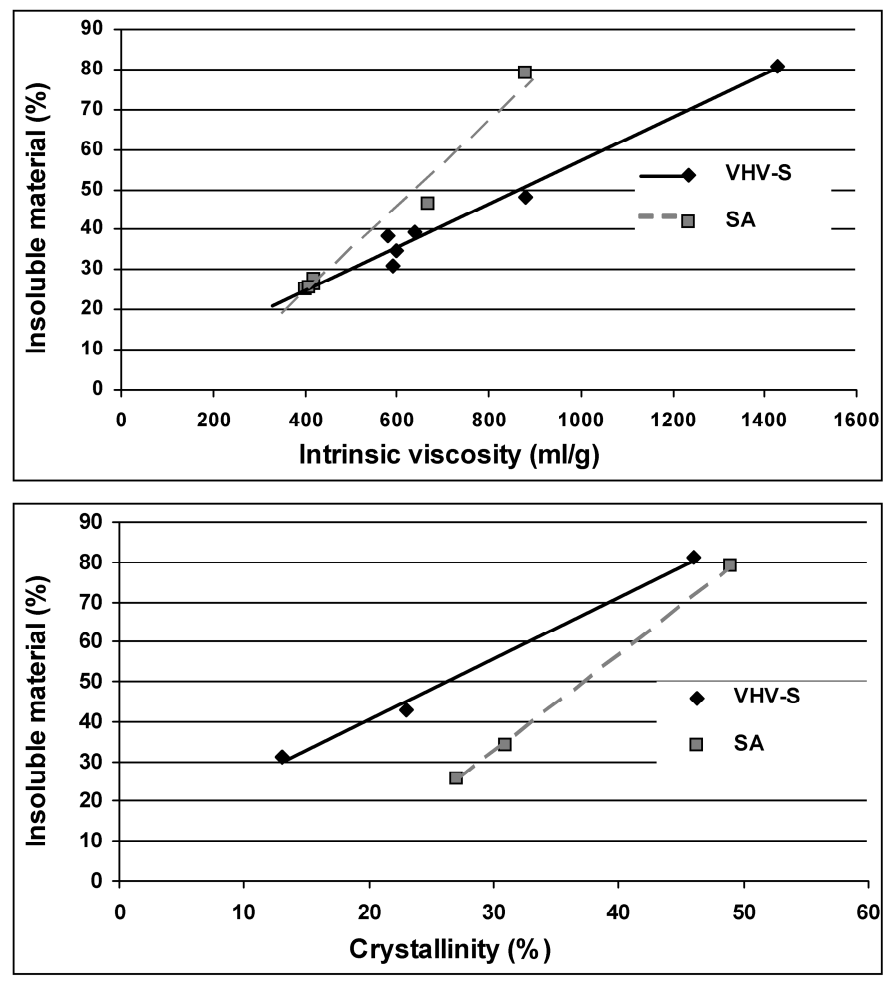

\section{Fig 9}




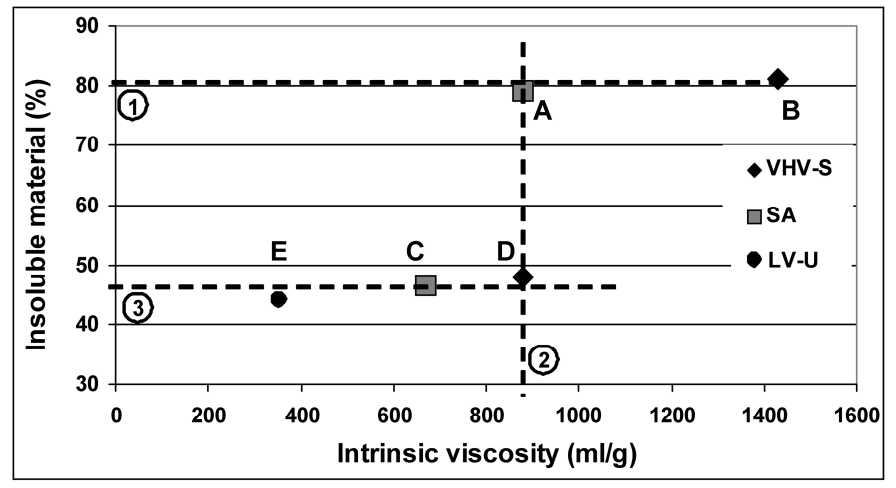

Fig 10 


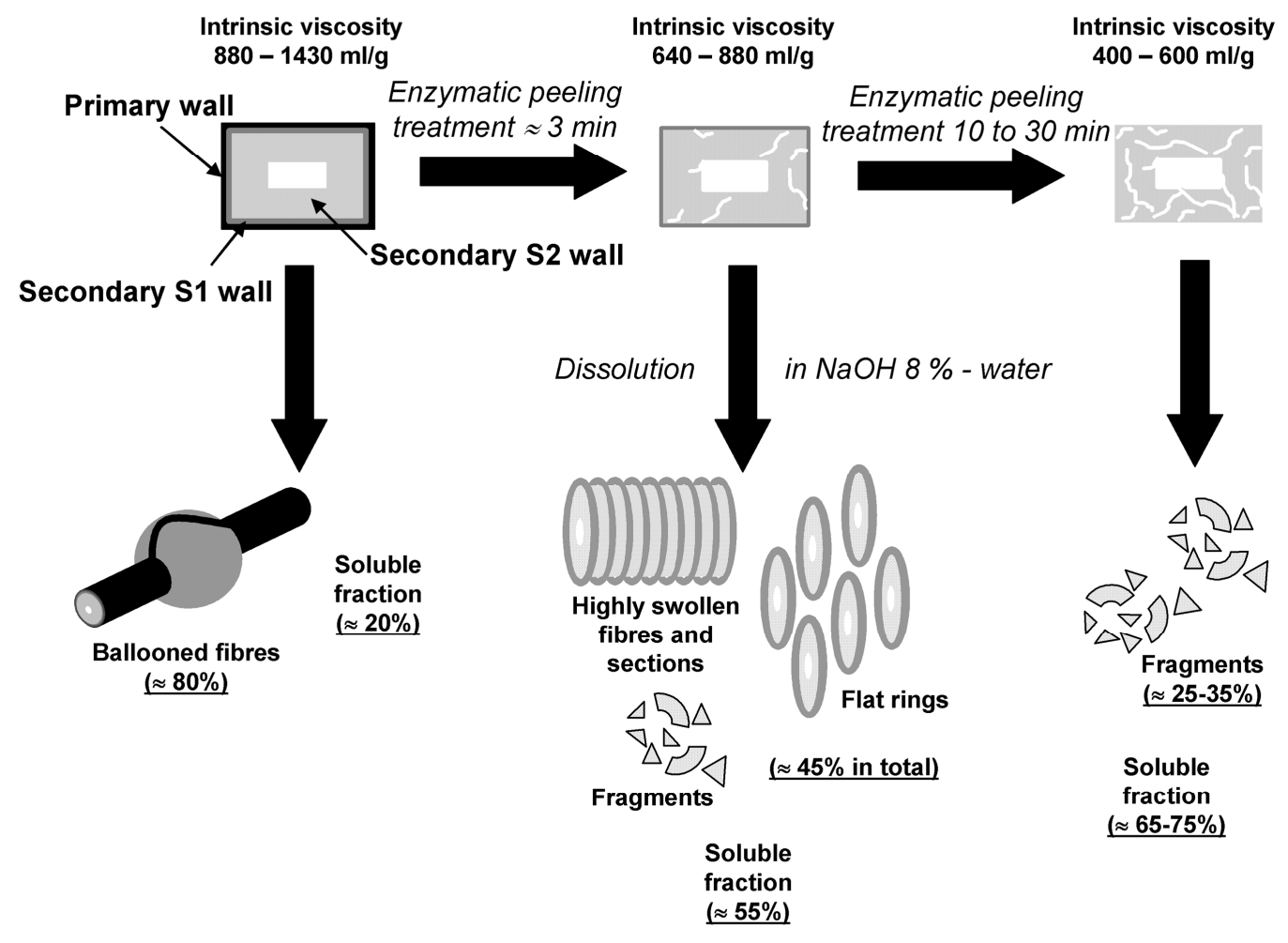

Fig 11 